\title{
Numerical Solution of a Class of Nonlinear System of Second-Order Boundary-Value Problems: a Fourth-Order Cubic Spline Approach
}

\section{Suheil A. Khuri and Ali M. Sayfy}

American University of Sharjah

Department of Mathematics and Statistics, P.O.Box 26666, Sharjah - UAE.

E-mail(corresp.): skhoury@aus.edu

E-mail: sayfy@aus.edu

Received June 22, 2014; revised August 19, 2015; published online September 15, 2015

\begin{abstract}
A cubic B-spline collocation approach is described and presented for the numerical solution of an extended system of linear and nonlinear second-order boundary-value problems. The system, whether regular or singularly perturbed, is tackled using a spline collocation approach constructed over uniform or non-uniform meshes. The rate of convergence is discussed theoretically and verified numerically to be of fourth-order. The efficiency and applicability of the technique are demonstrated by applying the scheme to a number of linear and nonlinear examples. The numerical solutions are contrasted with both analytical and other existing numerical solutions that exist in the literature. The numerical results demonstrate that this method is superior as it yields more accurate solutions.
\end{abstract}

Keywords: collocation method, finite element method, boundary value problem, differential equation.

AMS Subject Classification: 30C45, 31B30.

\section{Introduction}

In this paper, we study a generalized nonlinear system of second-order boundary value problems given by

$$
\begin{gathered}
a_{0}(x) u^{\prime \prime}+a_{1}(x) u^{\prime}+a_{2}(x) u+a_{3}(x) v^{\prime \prime}+a_{4}(x) v^{\prime}+a_{5}(x) v \\
+g_{1}\left(x, u, v, u^{\prime}, v^{\prime}\right)=f_{1}(x), \\
b_{0}(x) u^{\prime \prime}+b_{1}(x) u^{\prime}+b_{2}(x) u+b_{3}(x) v^{\prime \prime}+b_{4}(x) v^{\prime}+b_{5}(x) v \\
+g_{2}\left(x, u, v, u^{\prime}, v^{\prime}\right)=f_{2}(x) .
\end{gathered}
$$


The system is complimented with the following mixed boundary conditions:

$$
\begin{aligned}
& \gamma_{0} u(a)+\gamma_{1} v(a)+\gamma_{2} u^{\prime}(a)+\gamma_{3} v^{\prime}(a)=\eta_{0}, \\
& \xi_{0} u(a)+\xi_{1} v(a)+\xi_{2} u^{\prime}(a)+\xi_{3} v^{\prime}(a)=\eta_{1}, \\
& \lambda_{0} u(b)+\lambda_{1} v(b)+\lambda_{2} u^{\prime}(b)+\lambda_{3} v^{\prime}(b)=\eta_{2}, \\
& \mu_{0} u(b)+\mu_{1} v(b)+\mu_{2} u^{\prime}(b)+\mu_{3} v^{\prime}(b)=\eta_{3},
\end{aligned}
$$

where $a \leq x \leq b$. The functions $g_{1}(x), g_{2}(x), f_{1}(x), f_{2}(x)$ are continuous functions, and $a_{i}(x), b_{i}(x), i=0,1,2, \ldots 5$ are sufficiently smooth real-valued functions of $x$. Further, $\gamma_{i}, \xi_{i}, \lambda_{i}, \mu_{i}, \eta_{i}, i=0,1,2,3$ are constants. Normally, additional assumptions such as boundedness are imposed on the coefficients in order to guarantee the existence of a unique solution. For instance, in certain cases we need to require that $g_{1}, g_{2}$ are nonlinear functions in $u$ and $v$, where $u, v \in W_{2}^{3}[a, b]$, and $f_{i}-g_{i} \in W_{2}^{1}[a, b], i=1,2$.

In addition, we will study the following singularly perturbed nonlinear system of second-order boundary value problems:

$$
\begin{array}{r}
a_{0}(x ; \epsilon) u^{\prime \prime}+a_{1}(x) u^{\prime}+a_{2}(x) u+a_{3}(x) v^{\prime \prime}+a_{4}(x) v^{\prime} \\
+a_{5}(x) v+g_{1}\left(x, u, v, u^{\prime}, v^{\prime}\right)=f_{1}(x), \\
b_{0}(x) u^{\prime \prime}+b_{1}(x) u^{\prime}+b_{2}(x) u+b_{3}(x ; \epsilon) v^{\prime \prime}+b_{4}(x) v^{\prime} \\
+b_{5}(x) v+g_{2}\left(x, u, v, u^{\prime}, v^{\prime}\right)=f_{2}(x) .
\end{array}
$$

where $\epsilon \ll 1$. Such systems possess boundary layers and interior layers which pose some numerical difficulties and challenges.

In recent years, nonlinear equations [6] and systems of differential equations have been the focus of numerous articles that appear in the literature. This is due to their wide range of applicability in modeling problems that arise in engineering and other disciplines. Various special cases or versions of system (1.1)-(1.2), including ones that are singularly perturbed, have been of interest to many researchers whether in regard of proving existence or seeking numerical solutions. This extended system is one of the important kinds of nonlinear systems, and plays an important role in explaining many different phenomena. Special classes of this system as well as other classes have been tackled and solved in various ways. Cheng and Zhong [3] considered the existence of positive solutions for a second-order ordinary differential system, where the nonlinear term is superlinear in one equation and sublinear in the other equation. Geng and Cui [8] presented a method to obtain analytical and approximate solutions of linear and nonlinear systems of a class of second-order boundary-value problems. The analytical solution is represented in the form of series in the reproducing kernel space while the approximate solution $u_{n}(x)$ is obtained by the $n$-term intercept of the analytical solution and is proved to converge to the analytical solution. Valanarasu and Ramanujam [20] presented some numerical methods for singularly perturbed two-point boundary value problems for second order ordinary differential equations with two small parameters multiplying the derivatives. These methods are distinguished by the fact that, initial value problems and/or terminal value problems are constructed/deduced from the given boundary value problem and then solved by a fitted operator method. In [7], Dehghan and Saadatmandi employed the 
sinc-collocation method for solving a nonlinear system of second-order boundary value problems. Saadatmandi and J. Askari [18] presented the Chebyshev finite difference method, which can be regarded as a non-uniform finite difference scheme, for solving a nonlinear system of second-order boundary value problems. Their approach consists of reducing the problem to a set of algebraic equations. Saadatmandi et al. [17] proposed a homotopy perturbation method for solving a class of non-linear systems of second-order boundary-value problems. Khuri and Sayfy [9] manipulated a second-order spline collocation technique for the numerical solution of an extended system of second-order boundary-value problems. $\mathrm{Lu}$ [15] applied a variational iteration method to solve nonlinear system of second-order boundary value problems that is analogous to problem (1.1)-(1.2). The method yields solutions in convergent series forms with easily computable terms and the technique does not require any discretization, linearization or small perturbations. Bellew and O'Riordan [1], used a numerical method which is composed of an upwind finite difference operator on a piecewise-uniform Shishkin mesh to solve a coupled system of convection-diffusion problem with boundary layer. For further approaches to tackle nonlinear systems we refer the reader to $[14,16,17,18]$ and the references therein.

The ultimate goal of this article is to present and apply a cubic B-spline collocation strategy to obtain a numerical solution of the generalized nonlinear system (1.1)-(1.2). The theory of B-spline functions is an active field of approximation theory and is widely utilized for obtaining numerical solution of nonlinear problems. The B-spline curves have remarkable properties and features that make them fit for analysis and shape. The spline collocation method, which was first introduced by Christara and $\mathrm{Ng}$ [2] and [5], has been unified with an adaptive technique to solve the nonlinear system under consideration on uniform and non-uniform meshes via mesh redistribution [2] and manipulating an iterative scheme arising from Newton's method by mapping uniform node points to non-uniform ones such that the errors are reduced. This collocation approach has been employed by Khuri and Sayfy for the numerical solution of a spectrum of problems, including a boundary layer problem [12], a generalized nonlinear Klein-Gordon equation [10], a generalized parabolic problem subject to non-classical conditions [13], and Troesch's problem [11]. For further details and applications of the technique see $[4,5,9,10,11,12,13]$ and the references therein. The convergence analysis is deliberated and the method is verified to be of fourth-order rate of convergence which is then conformed numerically using the double-mesh principle. Applicability, performance and efficiency of the finite element collocation scheme is illustrated and tested on several test examples which represent special cases of the nonlinear system of differential equations. The depicted numerical results are compared with the exact solution and other available numerical methods. The outcomes results show the approach yields highly accurate results and converged fast using only a few number of mesh points.

The rest of this paper is organized as follows: in Section 2, the cubic B-spline collocation approach is described and presented for the numerical solution of the extended system of the second-order boundary-value problem. In section 3, 
the method is applied on a number of linear and nonlinear examples including one that is singularly perturbed with a boundary layer. The numerical results are compared with the exact solutions and other existing numerical solutions. Finally, a conclusion is given that summarizes the outcomes of the simulations.

\section{Cubic Spline Collocation Method}

In this section, we describe the finite element spline collocation scheme method and present the strategies on both a uniform and nonuniform meshes.

\subsection{Spline Collocation over a Uniform Mesh (SCU)}

Subsequent, we describe the cubic B-spline collocation approach on a uniform mesh for the numerical solution of the extended class of nonlinear system of the second-order mixed boundary-value problem given in (1.1)-(1.2). To construct the sought approximate solution, we consider the nodal points $x_{i}$ on the interval $[a, b]$ where

$$
a=x_{0}<x_{1}<\ldots<x_{n-1}<x_{n}=b .
$$

If the nodal points are equidistant from each other, then we have $x_{i}=a+$ $i h, i=0,1,2, \ldots, n$ where $h=\frac{b-a}{n}$ on the interval $[a, b]$. Let $\Psi(x)$ and $\Phi(x)$ be approximations that are approaching the exact solutions $u(x)$ and $v(x)$, respectively. These also satisfy the mixed boundary conditions (1.2) and are expressed as a linear combination of $n+3$ shape functions as follows:

$$
\Psi(x)=\sum_{i=-3}^{n-1} \alpha_{i} \psi_{i}(x), \quad \Phi(x)=\sum_{i=-3}^{n-1} \beta_{i} \psi_{i}(x) .
$$

The $\alpha_{i}$ 's and $\beta_{i}$ 's are unknown real coefficients and the $\psi_{i}(x)$ 's are the cubic B-splines functions given by:

$$
\psi_{i}(x)=\frac{1}{h^{3}} \begin{cases}\left(x-x_{i}\right)^{3}, & {\left[x_{i}, x_{i+1}\right],} \\ h^{3}+3 h^{2}\left(x-x_{i+1}\right)+3 h\left(x-x_{i+1}\right)^{2}-3\left(x-x_{i+1}\right)^{3}, & {\left[x_{i+1}, x_{i+2}\right],} \\ h^{3}+3 h^{2}\left(x_{i+3}-x\right)+3 h\left(x_{i+3}-x\right)^{2}-3\left(x_{i+3}-x\right)^{3}, & {\left[x_{i+2}, x_{i+3}\right],} \\ \left(x_{i+4}-x\right)^{3}, & {\left[x_{i+3}, x_{i+4}\right],} \\ 0, & \text { otherwise }\end{cases}
$$

where $h=x_{i+1}-x_{i}$. Considering the approximation function (2.1) and the cubic B-splines defined in (2.2), the required values of $\Psi\left(x_{j}\right)$ and its first and second derivatives with respect to the nodal points are identified in terms of $\alpha_{j}$ as:

$$
\begin{aligned}
\Psi\left(x_{j}\right) & =\alpha_{j-3}+4 \alpha_{j-2}+\alpha_{j-1} \\
\Psi^{\prime}\left(x_{j}\right) & =\frac{3}{h}\left(\alpha_{j-3}-\alpha_{j-1}\right), \quad \Psi^{\prime \prime}\left(x_{j}\right)=\frac{6}{h^{2}}\left(\alpha_{j-3}-2 \alpha_{j-2}+\alpha_{j-1}\right) .
\end{aligned}
$$


The values of $\Phi\left(x_{j}\right)$ are determined similarly as in (2.3) with $\alpha_{j}$ replaced by $\beta_{j}$. Substituting the approximate solutions $\Phi(x)$ and $\Psi(x)$, given by $(2.1)$, into equations (1.1) we have

$$
\begin{aligned}
& \sum_{i=-3}^{n-1} \alpha_{i}\left[a_{0}\left(x_{j}\right) \psi_{i}^{\prime \prime}\left(x_{j}\right)+a_{1}\left(x_{j}\right) \psi_{i}^{\prime}\left(x_{j}\right)+a_{2}\left(x_{j}\right) \psi_{i}\left(x_{j}\right)\right] \\
& \quad+\sum_{i=-3}^{n-1} \beta_{i}\left[a_{3}\left(x_{j}\right) \psi_{i}^{\prime \prime}\left(x_{j}\right)+a_{4}\left(x_{j}\right) \psi_{i}^{\prime}\left(x_{j}\right)+a_{5}\left(x_{j}\right) \psi_{i}\left(x_{j}\right)\right] \\
& +g_{1}\left(x_{j}, \sum_{i=-3}^{n-1} \alpha_{i} \psi_{i}\left(x_{j}\right), \sum_{i=-3}^{n-1} \beta_{i} \psi_{i}\left(x_{j}\right), \sum_{i=-3}^{n-1} \alpha_{i} \psi_{i}^{\prime}\left(x_{j}\right), \sum_{i=-3}^{n-1} \beta_{i} \psi_{i}^{\prime}\left(x_{j}\right)\right)=f_{1}\left(x_{j}\right), \\
& \quad j=0,1,2, \ldots, n .
\end{aligned}
$$

For the second differential equation we obtain

$$
\begin{aligned}
& \sum_{i=-3}^{n-1} \alpha_{i}\left[b_{0}\left(x_{j}\right) \psi_{i}^{\prime \prime}\left(x_{j}\right)+b_{1}\left(x_{j}\right) \psi_{i}^{\prime}\left(x_{j}\right)+b_{2}\left(x_{j}\right) \psi_{i}\left(x_{j}\right)\right] \\
& \quad+\sum_{i=-3}^{n-1} \beta_{i}\left[b_{3}\left(x_{j}\right) \psi_{i}^{\prime \prime}\left(x_{j}\right)+b_{4}\left(x_{j}\right) \psi_{i}^{\prime}\left(x_{j}\right)+b_{5}\left(x_{j}\right) \psi_{i}\left(x_{j}\right)\right] \\
& +g_{2}\left(x_{j}, \sum_{i=-3}^{n-1} \alpha_{i} \psi_{i}\left(x_{j}\right), \sum_{i=-3}^{n-1} \beta_{i} \psi_{i}\left(x_{j}\right), \sum_{i=-3}^{n-1} \alpha_{i} \psi_{i}^{\prime}\left(x_{j}\right), \sum_{i=-3}^{n-1} \beta_{i} \psi_{i}^{\prime}\left(x_{j}\right)\right)=f_{2}\left(x_{j}\right), \\
& \quad j=0,1,2, \ldots, n .
\end{aligned}
$$

The above system consists of $2 n+2$ equations in $2 n+6$ unknowns. The mixed boundary conditions in (1.2) yield the following four conditions:

$$
\begin{aligned}
& \sum_{i=-3}^{n-1} \alpha_{i}\left(\gamma_{0} \psi_{i}\left(x_{j}\right)+\gamma_{2} \psi_{i}^{\prime}\left(x_{j}\right)\right)+\sum_{i=-3}^{n-1} \beta_{i}\left(\gamma_{1} \psi_{i}\left(x_{j}\right)+\gamma_{3} \psi_{i}^{\prime}\left(x_{j}\right)\right)=\eta_{0}, j=0, \\
& \sum_{i=-3}^{n-1} \alpha_{i}\left(\xi_{0} \psi_{i}\left(x_{j}\right)+\xi_{2} \psi_{i}^{\prime}\left(x_{j}\right)\right)+\sum_{i=-3}^{n-1} \beta_{i}\left(\xi_{1} \psi_{i}\left(x_{j}\right)+\xi_{3} \psi_{i}^{\prime}\left(x_{j}\right)\right)=\eta_{1}, j=0, \\
& \sum_{i=-3}^{n-1} \alpha_{i}\left(\lambda_{0} \psi_{i}\left(x_{j}\right)+\lambda_{2} \psi_{i}^{\prime}\left(x_{j}\right)\right)+\sum_{i=-3}^{n-1} \beta_{i}\left(\lambda_{1} \psi_{i}\left(x_{j}\right)+\lambda_{3} \psi_{i}^{\prime}\left(x_{j}\right)\right)=\eta_{0}, j=n, \\
& \sum_{i=-3}^{n-1} \alpha_{i}\left(\mu_{0} \psi_{i}\left(x_{j}\right)+\mu_{2} \psi_{i}^{\prime}\left(x_{j}\right)\right)+\sum_{i=-3}^{n-1} \beta_{i}\left(\mu_{1} \psi_{i}\left(x_{j}\right)+\mu_{3} \psi_{i}^{\prime}\left(x_{j}\right)\right)=\eta_{0}, j=n .
\end{aligned}
$$

The values of $\Psi, \Psi^{\prime}$ and $\Psi^{\prime \prime}$ at the nodal points $x_{j}, j=0,1, \ldots, n$ are determined from equation (2.3) and similarly for the values of $\Phi$.

The system of equations in (2.4), (2.6), and (2.8) can be written in matrix form as follows:

$$
\mathrm{C}_{1} \mathbf{d}+\mathrm{M}_{1} \mathbf{e}+\mathrm{g}_{1}=\mathbf{f}_{1}
$$


where

and

$$
\mathbf{C}_{1}=\left[\begin{array}{ccccccc}
r_{-1} & s_{-1} & p_{-1} & 0 & 0 & \ldots & 0 \\
r_{0} & s_{0} & p_{0} & 0 & 0 & \ldots & 0 \\
0 & r_{1} & s_{1} & p_{1} & 0 & \ldots & 0 \\
. & . & . & . & . & . & . \\
. & . & . & . & . & . & . \\
0 & 0 & 0 & \ldots & r_{n} & s_{n} & p_{n} \\
0 & 0 & 0 & \ldots & r_{n+1} & s_{n+1} & p_{n+1}
\end{array}\right]
$$

$$
\mathbf{M}_{1}=\left[\begin{array}{ccccccc}
q_{-1} & w_{-1} & z_{-1} & 0 & 0 & \ldots & 0 \\
q_{0} & w_{0} & z_{0} & 0 & 0 & \ldots & 0 \\
0 & q_{1} & w_{1} & z_{1} & 0 & \ldots & 0 \\
\cdot & \cdot & \cdot & \cdot & . & . & . \\
. & . & . & . & . & . & \cdot \\
0 & 0 & 0 & \ldots & q_{n} & w_{n} & z_{n} \\
0 & 0 & 0 & \ldots & q_{n+1} & w_{n+1} & z_{n+1}
\end{array}\right] \text {. }
$$

The above matrices have dimension $(n+3) \times(n+3)$. Here

$$
\begin{aligned}
& r_{-1}=\gamma_{0}-\frac{3}{h} \gamma_{2}, \quad s_{-1}=4 \gamma_{0}, \quad p_{-1}=\gamma_{0}+\frac{3}{h} \gamma_{2}, \\
& r_{n+1}=\lambda_{0}-\frac{3}{h} \lambda_{2}, \quad s_{n+1}=4 \lambda_{0}, \quad p_{n+1}=\lambda_{0}+\frac{3}{h} \lambda_{2}, \\
& r_{j}=\frac{6 a_{0 j}}{h^{2}}+\frac{3 a_{1 j}}{h}+a_{2 j}, \quad s_{j}=-\frac{12 a_{0 j}}{h^{2}}+4 a_{2 j}, \quad p_{j}=\frac{6 a_{0 j}}{h^{2}}-\frac{3 a_{1 j}}{h}+a_{2 j}, \\
& j=0,1, \ldots n,
\end{aligned}
$$

given that $a_{0 j}=a_{0}\left(x_{j}\right), a_{1 j}=a_{1}\left(x_{j}\right), a_{2 j}=a_{2}\left(x_{j}\right)$, where $x_{j}=a+j h$. Further

$$
\begin{aligned}
& q_{j}=\frac{6 a_{3 j}}{h^{2}}+\frac{3 a_{4 j}}{h}+a_{5 j}, \quad w_{j}=-\frac{12 a_{3 j}}{h^{2}}+4 a_{5 j}, \quad z_{j}=\frac{6 a_{3 j}}{h^{2}}-\frac{3 a_{4 j}}{h}+a_{5 j}, \\
& j=0,1, \ldots n,
\end{aligned}
$$

given that $a_{3 j}=a_{3}\left(x_{j}\right), a_{4 j}=a_{4}\left(x_{j}\right), a_{5 j}=a_{5}\left(x_{j}\right)$, where $x_{j}=a+j h$.

The vectors $\mathbf{g}_{1}^{\mathbf{T}}, \mathbf{d}^{\mathbf{T}}$, and $\mathbf{e}^{\mathbf{T}}$ are given by

$$
\begin{aligned}
& \mathbf{g}_{\mathbf{1}}^{\mathbf{T}}=\left[0, g_{12}, g_{13}, \ldots, g_{1(n+2)}, 0\right], \mathbf{d}^{\mathbf{T}}=\left[\alpha_{-3}, \alpha_{-2}, \alpha_{-1}, \alpha_{0}, \ldots, \alpha_{n-2}, \alpha_{n-1}\right], \\
& \mathbf{e}^{\mathbf{T}}=\left[\beta_{-3}, \beta_{-2}, \beta_{-1}, \beta_{0}, \ldots, \beta_{n-3}, \beta_{n-2}, \beta_{n-1}\right] \\
& \mathbf{f}_{\mathbf{1}}^{\mathbf{T}}=\left[\eta_{0}, f_{1}\left(x_{0}\right), f_{1}\left(x_{1}\right), f_{1}\left(x_{2}\right), \ldots, f_{1}\left(x_{n-1}\right), f_{1}\left(x_{n}\right), \eta_{2}\right],
\end{aligned}
$$

where

$$
\begin{aligned}
g_{1 j}= & g_{1}\left(x_{j}, \alpha_{j-5}+4 \alpha_{j-4}+\alpha_{j-3}, \beta_{j-5}+4 \beta_{j-4}+\beta_{j-3}, \frac{-3}{h} \alpha_{j-5}+\frac{3}{h} \alpha_{j-3},\right. \\
& \left.\frac{-3}{h} \beta_{j-5}+\frac{3}{h} \beta_{j-3}\right), \quad j=2,3, \ldots, n+2 .
\end{aligned}
$$

In a similar fashion, the system (2.5), (2.7), and (2.9) can be written in matrix form as follows:

$$
\mathrm{C}_{2} \mathrm{~d}+\mathrm{M}_{2} \mathrm{e}+\mathrm{g}_{2}=\mathrm{f}_{2}
$$


The matrices $\mathbf{C}_{\mathbf{2}}, \mathbf{M}_{\mathbf{2}}, \mathbf{g}_{\mathbf{2}}$ and $\mathbf{f}_{\mathbf{2}}$ are similar to $\mathbf{C}_{\mathbf{1}}, \mathbf{M}_{\mathbf{1}}, \mathbf{g}_{\mathbf{1}}$ and $\mathbf{f}_{\mathbf{1}}$ except that the functions $a_{i}, i=0,1, \ldots, 5$ and $f_{1}$ are replaced by the functions $b_{i}, i=$ $0,1, \ldots, 5$ and $f_{2}$, respectively. As for the boundary conditions, we replace $\eta_{0}, \eta_{2}$ in $\mathbf{f}_{\mathbf{1}}$ by $\eta_{1}, \eta_{3}$ to obtain $\mathbf{f}_{\mathbf{2}}$.

Systems (2.10) and (2.11) can be combined as one system as follows:

$$
\left[\begin{array}{l}
\mathrm{C}_{1} \\
\mathrm{C}_{2}
\end{array}\right] \mathrm{d}+\left[\begin{array}{l}
\mathrm{M}_{1} \\
\mathrm{M}_{2}
\end{array}\right] \mathrm{e}+\left[\begin{array}{l}
\mathrm{g}_{1} \\
\mathrm{~g}_{2}
\end{array}\right]=\left[\begin{array}{l}
\mathrm{f}_{1} \\
\mathrm{f}_{2}
\end{array}\right]
$$

The system of equations given in (2.12) is solved using the computer algebra system Maple.

\subsection{Spline Collocation over a Non-uniform Mesh (SCN)}

Next, we will briefly present and describe the spline collocation method, based on non-uniform meshes, for obtaining a numerical solution for the system of differential equations (1.1) subject to the mixed boundary conditions (1.2). For completeness and more detailed analysis, we refer the reader to the paper by Christara and $\mathrm{Ng}[4]$ that includes a thorough discussion of the scheme.

To construct an approximate solution on $[a, b]$, we first consider a mesh with uniform node points $x_{0}<x_{1}<\ldots<x_{N}$, where $x_{i}=a+i h, i=0,1,2, . ., N ; h=$ $(b-a) / N$. Various transformations and grading functions are available [2] for the re-distribution of the nodes on $[0,1]$. We need to select or construct a strictly increasing bijective function that maps the uniform nodes $\left\{x_{i}\right\}$ to nonuniform meshes with nodes $\left\{w_{i}\right\}$ tailored suitably in order to reduce the error. The intent of these mappings is to create finer meshes near the ends in case of existence of singularities or boundary layers. To have more nodes near one endpoint we use the following mappings redistribution functions:

$$
z_{1}(x)=(b-a)\left(1-\frac{(1+k)^{1-\frac{x_{i}-a}{b-a}}-1}{k}\right)+a,
$$

and

$$
z_{2}(x)=(b-a)\left(\frac{(1+k)^{\frac{x_{i}-a}{b-a}}-1}{k}\right)+a .
$$

The transformation functions $z_{1}(x)$ and $z_{2}(x)$ redistribute the nodes on $[a, b]$ with more nodes near $x=a$ and $x=b$, respectively, as $k$ increases. These are convenient if a boundary layer is located at one end only.

For a problem with layers at both ends of $[0,1]$, we use the following Chebyshev-Gauss-Lobatto points (Lobatto points in short):

$$
x_{i}=\frac{1}{2}\left(1-\cos \left(\frac{i}{N} \pi\right)\right) .
$$

For a finer mesh near the boundaries and coarser mesh in the regular region, we use the transformation

$$
z_{i}=(1-\tau)\left(3 x_{i}^{2}-2 x_{i}^{3}\right)+\tau x_{i}
$$


where $\tau$ is the adjustment parameter. In case a redistribution is required on the interval $[a, b]$, a simple linear transformation can be used in order to map the interval $[0,1]$ onto $[a, b]$. The mesh selection strategy for a system with layers depends on the thickness of the layers, which can be estimated by a Shishkin-like formula.

Next, we describe the implementation of the (SCN) for the numerical solution of the subsequent linear boundary value problem:

$$
L[u(x)] \equiv r(x) u^{\prime \prime}+p(x) u^{\prime}+q(x) u=g(x),
$$

where $x \in \Omega=(a, b)$. The equation is subject to the the boundary conditions:

$$
u(a)=\nu_{0}, \quad u(b)=\nu_{1} .
$$

We approximate the exact solution $u$ by a linear combination of spline functions, $\Psi(x)$, as follows:

$$
\Psi(x)=\sum_{i=-3}^{n-1} c_{i} \psi_{i}(x),
$$

where $\Psi_{i}(x)$ is a nonuniform spline function defined by

$$
\psi_{i}(x)=\left\{\begin{array}{l}
\frac{\left(x-w_{i}\right)^{3}}{w_{i, 3} w_{i, 2} w_{i, 1}}, \quad w_{i} \leq x \leq w_{i+1}, \\
\frac{x-w_{i}}{w_{i, 3}}\left[\frac{\left(x-w_{i}\right)\left(w_{i+2}-x\right)}{w_{i, 2}, ; w_{i+1,1}}+\frac{\left(x-w_{i+1}\right)\left(w_{i+3}-x\right)}{w_{i+1,2} w_{i+1,1}}\right] \\
\quad+\frac{\left(x-w_{i+1}\right)^{2}\left(w_{i+4}-x\right)}{w_{i+1,3} w_{i+1,2} w_{i+1,1}}, \quad w_{i+1} \leq x \leq w_{i+2}, \\
\frac{w_{i+4}-x}{w_{i+1,3}}\left[\frac{\left(x-w_{i+1}\right)\left(w_{i+3}-x\right)}{w_{i+1,2} w_{i+2,1}}+\frac{\left(x-w_{i+2}\right)\left(w_{i+4}-x\right)}{w_{i+2,2} w_{i+2,1}}\right] \\
\quad+\frac{\left(x-w_{i}\right)\left(w_{i+3}-x\right)^{2}}{w_{i, 3} w_{i+1,2} w_{i+2,1}}, \quad w_{i+2} \leq x \leq w_{i+3}, \\
\frac{\left(w_{i+4}-x\right)^{3}}{w_{i+1,3} w_{i+2,2} w_{i+3,1}}, \quad w_{i+3} \leq x \leq w_{i+4} \\
0, \quad \text { otherwise, }
\end{array}\right.
$$

where $w_{i, j}=w_{i+j}-w_{i}$. The constant coefficients $\left\{c_{i}\right\}$ in (2.18) are the solutions of the following system of linear equations:

$$
\begin{aligned}
& \Psi\left(w_{0}\right)=\gamma_{0}, \quad \Psi\left(w_{N}\right)=\gamma_{1}, \\
& L\left[\Psi\left(w_{j}\right)\right]=g\left(w_{j}\right)-\mathcal{L}_{p}\left[\Psi\left(w_{j}\right)\right], \quad j=0,1, \ldots, N,
\end{aligned}
$$

where the operator $\mathcal{L}_{p}$, which gives optimum order [4], is defined by

$$
\begin{aligned}
\mathcal{L}_{p}\left[\Psi\left(w_{0}\right)\right] & =\frac{r\left(w_{0}\right) h_{0}}{24}\left(5 h_{0}-4 h_{1}+h_{2}\right) \frac{\left(h_{0}+h_{1}\right) \Gamma\left[\Psi^{\prime \prime}\left(w_{1}\right)\right]-h_{0} \Gamma\left[\Psi^{\prime \prime}\left(w_{2}\right)\right]}{h_{1}}, \\
\mathcal{L}_{p}\left[\Psi\left(w_{N}\right)\right] & =\frac{r\left(w_{N}\right)}{24} h_{N-1}\left(5 h_{N-1}-4 h_{N-2}+h_{N-3}\right) \\
\times & \frac{\left(h_{N-1}+h_{N-2}\right) \Gamma\left[\Psi^{\prime \prime}\left(w_{N-1}\right)\right]-h_{N-1} \Gamma\left[\Psi^{\prime \prime}\left(w_{N-2}\right)\right]}{h_{N-2}}, \\
\mathcal{L}_{p}\left[\Psi\left(w_{j}\right)\right] & =\frac{r\left(w_{j}\right)}{12} h_{j} h_{j-1} \Gamma\left[\Psi^{\prime \prime}\left(w_{j}\right)\right], \quad j=1,2,3, \ldots, N-1,
\end{aligned}
$$


where

$$
\Gamma\left[\Psi\left(w_{j}\right)\right]=\frac{2 h_{j} \Psi\left(w_{j-1}\right)-2\left(h_{j-1}+h_{j}\right) \Psi\left(w_{j}\right)+2 h_{j-1} \Psi\left(w_{j+1}\right)}{h_{j-1}\left(h_{j-1}+h_{j}\right) h_{j}}
$$

and $h_{j}=w_{j+1}-w_{j}$. Note that $\Gamma\left[\Psi^{\prime \prime}\left(w_{j}\right)\right]$ approximates the fourth derivative of $\psi$ at $w_{j}$. For a uniform partition $\Gamma\left[S\left(w_{j}\right)\right]$ reduces to the three-point central difference approximation formula for the second derivative, namely,

$$
\Gamma\left[\Psi\left(w_{j}\right)\right]=\frac{\Psi\left(w_{j-1}\right)-2 \Psi\left(w_{j}\right)+\Psi\left(w_{j+1}\right)}{h^{2}} .
$$

Finally we determine the values of the spline functions and their first and second derivatives at the nodes $w_{i+1}, w_{i+2}, w_{i+3}$. Upon denoting

$$
\Psi_{i}^{(r)}=\left[\Psi_{i}^{(r)}\left(w_{i+1}\right), \Psi_{i}^{(r)}\left(w_{i+2}\right), \Psi_{i}^{(r)}\left(w_{i+3}\right)\right]
$$

for $r=0,1,2$, the system (2.20) simplifies to the following $N+3$ linear system:

$$
\begin{aligned}
& \Psi_{-3,3} c_{-3}+\Psi_{-2,2} c_{-2}+\Psi_{-1,1} c_{-1}=\gamma_{0}, \\
& \Psi_{N-3,3} c_{N-3}+\Psi_{N-2,2} c_{N-2}+\Psi_{N-1,1} c_{N-1}=\gamma_{1}, \\
& r\left(w_{j}\right)\left\{\Psi_{j-3,3}^{\prime \prime} c_{j-3}+\Psi_{j-2,2}^{\prime \prime} c_{j-2}+\Psi_{j-1,1}^{\prime \prime} c_{j-1}\right\}+p\left(w_{j}\right)\left\{\Psi_{j-3,3}^{\prime} c_{j-3}\right. \\
& \left.\quad+\Psi_{j-2,2}^{\prime} c_{j-2}+\Psi_{j-1,1}^{\prime} c_{j-1}\right\}+\left(q\left(w_{j}\right) I-\mathcal{L}_{p}\right)\left[\Psi_{j-3,3} c_{j-3}\right. \\
& \left.\quad+\Psi_{j-2,2} c_{j-2}+\Psi_{j-1,1} c_{j-1}\right]=g\left(w_{j}\right), \quad \text { for } j=1,2, \ldots, N-1,
\end{aligned}
$$

where $I$ is the identity operator. For nonlinear systems (1.1), the system is rearranged after replacing $g_{1}$ and $g_{2}$ by the following Taylor expansions:

$$
\begin{aligned}
g_{i}\left(x, u_{m}, v_{m}, u_{m}^{\prime}, v_{m}^{\prime}\right) & =g_{i}\left(x, u_{m-1}, v_{m-1}, u_{m-1}^{\prime}, v_{m-1}^{\prime}\right) \\
& +\left(u_{m}-u_{m-1}\right) \frac{\partial}{\partial u} g_{i}\left(x, u_{m-1}, v_{m-1}, u_{m-1}^{\prime}, v_{m-1}^{\prime}\right) \\
& +\left(v_{m}-v_{m-1}\right) \frac{\partial}{\partial v} g_{i}\left(x, u_{m-1}, v_{m-1}, u_{m-1}^{\prime}, v_{m-1}^{\prime}\right) \\
& +\left(u_{m}^{\prime}-u_{m-1}^{\prime}\right) \frac{\partial}{\partial u^{\prime}} g_{i}\left(x, u_{m-1}, v_{m-1}, u_{m-1}^{\prime}, v_{m-1}^{\prime}\right) \\
& +\left(v_{m}^{\prime}-v_{m-1}^{\prime}\right) \frac{\partial}{\partial v^{\prime}} g_{i}\left(x, u_{m-1}, v_{m-1}, u_{m-1}^{\prime}, v_{m-1}^{\prime}\right)
\end{aligned}
$$

for $i=1,2$.

\subsection{Convergence of the Adaptive Method}

In this subsection, we discuss convergence of the cubic spline collocation method, in particular it will shown that the scheme has order four rate of convergence.

Given a function $w(x)$, let $u_{\Delta_{w}}^{3}$ denote the cubic spline space with respect to a partition $\Delta_{w}$. Basically, we assume that $u_{\Delta[1]}^{3}$ is a cubic spline satisfying the following equations:

$$
\begin{aligned}
& L u_{\Delta[1]}^{3}=g \text { in } \Delta_{w}, \\
& B u_{\Delta[1]}^{3}=\gamma \text { on } T_{w \mathbf{B}},
\end{aligned}
$$


and the cubic spline $u_{\Delta}^{3}$ satisfies:

$$
\begin{aligned}
& L u_{\Delta}^{3}=g-\mathcal{L}_{p} u_{\Delta[1]}^{3} \text { in } \Delta_{w}, \\
& B u_{\Delta}^{3}=\gamma \text { on } T_{w \mathbf{B}} .
\end{aligned}
$$

The operator $L$ is given in $(2.16), \mathcal{L}_{p}$ defined in $(2.21), B$ are any specified mixed boundary conditons, and finally $T_{w \mathbf{B}}$ is the set of boundary collocation points with respect to $w$. The next two theorems, proven by Christara and $\mathrm{Ng}[5]$, show that the rate of convergence is of order four.

Theorem 1. Assume

(A1) the coefficients $p$ and $q$, and the right-side $g$ are $\mathbf{C}[\Omega]$,

(A2) the $B V P L[u]=g, B u=0$ has a unique solution,

(A3) the $B V P u^{\prime \prime}=0, B u=0$ has a unique solution,

(A4) $u \in \mathbf{C}^{\mathbf{6}}[\bar{\Omega}], w(x): \bar{\Omega} \rightarrow \bar{\Omega}$ is a bijective map in $\mathbf{C}^{3}$, with $w^{\prime}(x)>0, \forall x \in$ $\bar{\Omega}, w^{-1} \in \mathbf{C}^{\mathbf{1}}[\bar{\Omega}]$,

then $u_{\Delta}^{3} \in S_{\Delta_{w}}^{3}$ defined by (2.22) exists, is unique, and satisfies the global error estimates

$$
\left\|\left(u-u_{\Delta[1]}^{3}\right)^{(k)}\right\|_{\infty}=\mathcal{O}\left(h^{2}\right), \quad k=0,1,2, \quad\left\|\left(u-u_{\Delta[1]}^{3}\right)^{(3)}\right\|_{\infty}=\mathcal{O}(h),
$$

and the local error estimates

$$
\left|\left(u-u_{\Delta[1]}^{3}\right)^{(3)}\left(w_{i}\right)\right|_{\infty}=\mathcal{O}\left(h^{2}\right), \quad i=1,2, \ldots, N .
$$

Define the Gaussian points $\delta_{i j}=x_{i}-\lambda_{j} h ; j=1,2, \quad i=1, \ldots, N$, where $\lambda_{1}=(3-\sqrt{3}) / 6$ and $\lambda_{2}=(3+\sqrt{3}) / 6$. Let $w(x): \bar{\Omega} \rightarrow \bar{\Omega}$ be a bijective function in $C^{3}$ with $w^{\prime}(x)>0$ for all $x$ and let $w_{i}$ be the set of collocation points.

Theorem 2. Under the assumptions of Theorem 1, and the assumption that $u^{\prime \prime}-u_{\Delta[1]}^{3^{\prime \prime}}$ has a smooth expansion at the collocation points $u_{\Delta}^{3} \in S_{\Delta_{w}}^{3}$ defined by (2.23) exists, is unique, and satisfies the global error estimates

$$
\left\|\left(u-u_{\Delta}^{3}\right)^{(k)}\right\|_{\infty}=\mathcal{O}\left(h^{4-k}\right), \quad k=0,1,2,3
$$

and the local error estimates

$$
\mid \begin{aligned}
& \left(u-u_{\Delta}^{3}\right)^{\prime}(x) \mid=\mathcal{O}\left(h^{4}\right) \quad \text { for } x=s_{i} \text { and } w_{i} \\
& \left(u-u_{\Delta}^{3}\right)^{\prime \prime}\left(\sigma_{i j}\right)\left|=\mathcal{O}\left(h^{3}\right), \quad\right|\left(u-u_{\Delta}^{3}\right)^{\prime \prime \prime}\left(w_{i}\right) \mid=\mathcal{O}\left(h^{2}\right) .
\end{aligned}
$$

All the coefficients in system (1.1) are all assumed to be continuous on the given domain. As for the nonlinear terms $g_{1}$ and $g_{2}$, they will be linearized using Newton's method and therefore continuity in $u$ follows and consequently 
assumption $A 1$ of Theorem 1 is satisfied. Necessary assumptions on the coefficients are imposed to guarantee the existence of a unique solution of problem (1.1)-(1.2) and thus condition A2 holds. The mixed boundary conditions chosen can easily warantee that the solution of $u^{\prime \prime}=0$ subject to these given conditions is unique, thus assumption $A 3$ of Theorem 2 holds. This assumption is also valid for the solution $v$, so basically in our previous and upcoming discussion for convergence we intend to the check the validity of the assumptions to $v$ as well, that is, we need to apply the theorem to the vector solution $\mathbf{U}=\left(\begin{array}{l}u \\ v\end{array}\right)$. In the test examples, we have used the mapping redistribution functions given in $(2.13),(2.14)$ and $(2.15)$. The first two transformations are clearly bijective maps in $\mathbf{C}^{3}$ that take the interval $[a, b]$ onto $[a, b]$. Further, it can be easily shown that $w^{\prime}(x)>0$ for our choice $k>0$ and the inverse function $w^{-1}$ can be easily computed and shown that $w^{-1} \in \mathbf{C}^{\mathbf{1}}[a, b]$. We will assume that the solution of (1.1)-(1.2) is smooth enough so that $u \in \mathbf{C}^{\mathbf{6}}[0,1]$, that is, the last assumption $A_{4}$ is satisfied and so the conclusion of Theorem 1 follows. As for the Chebyshev-Gauss-Lobatto function, we have

$$
w_{i}^{\prime}=6(1-\tau) z_{i}\left(1-z_{i}\right)+\tau .
$$

Since $z_{i} \in[0,1]$ and $\tau \in[0,1]$, thus $w_{i}^{\prime}>0$. The inverse function theorem implies that $w_{i}^{-1} \in \mathbf{C}^{\mathbf{1}}[0,1]$. The assumptions of Theorem 2 are same as Theorem 1 in additon to the assumption that $u^{\prime \prime}-u_{\Delta[1]}^{3^{\prime \prime}}$ has a smooth expansion at the collocation points $u_{\Delta}^{3} \in S_{\Delta_{w}}^{3}$, which is true for this case. Consequently the conclusion of Theorem 2 follows as well.

\section{Numerical Examples}

In this section, the cubic spline collocation scheme is applied to a number of linear and nonlinear systems of equations that are special cases of the problem under consideration, namely equation (1.1)-(1.2). The five test examples that are examined appear in the literature and include: a linear system, two nonlinear systems, and two singularly perturbed reaction-diffusion and convectiondiffusion systems of equations. The purpose of the simulations is to corroborate the applicability, efficiency and high accuracy of the proposed method. It is verified numerically that the approach has optimum rate of convergence of order 4 as is reported in the tables. This rate has been obtained by the following log ratio formula:

$$
p \approx \frac{\ln \left(\operatorname{Err}\left(n_{i}\right)\right)-\ln \left(\operatorname{Err}\left(n_{i+1}\right)\right)}{\ln \left(\frac{b-\sigma}{n_{i}}\right)-\ln \left(\frac{b-\sigma}{n_{i+1}}\right)},
$$

where $\operatorname{Err}(n)=\max _{i}\left|y_{i}^{n}-y\left(x_{i}\right)\right|$. Here $y_{i}^{n}$ is the numerical value at $x_{i}$ using $n$ mesh intervals while $y\left(x_{i}\right)$ is the exact value at $x_{i}$.

Example 1. Consider the following second-order linear system:

$$
\left\{\begin{array}{l}
u^{\prime \prime}(x)+u^{\prime}(x)+x u(x)+v^{\prime}(x)+2 x v(x)=f_{1}(x), \\
2 u^{\prime}(x)+x^{2} u(x)+v^{\prime \prime}(x)+v(x)=f_{2}(x), \\
u(0)=u(1)=0, \quad v(0)=v(1)=0
\end{array}\right.
$$


where $0 \leq x \leq 1$,

$$
\begin{aligned}
& f_{1}(x)=-2(1+x) \cos x+\pi \cos \pi x+2 x \sin \pi x+\left(4 x-2 x^{2}-4\right) \sin x, \\
& f_{2}(x)=-4(x-1) \cos x-2\left(2-x^{2}+x^{3}\right) \sin x-\left(\pi^{2}-1\right) \sin \pi x
\end{aligned}
$$

The exact solution of $(3.1)$ is $u(x)=2(1-x) \sin x$ and $v(x)=\sin \pi x$.

The maximum error resulting from the numerical solution obtained by the cubic spline collocation method, using $N=8,16,32,64$ nodal points on a uniform mesh, are reported in Table 1 and are compared with the results obtained by Geng [8]. Obviously, the maximum errors acquired by the spline collocation approach (SCA) are notably smaller than those given in [8]. It is worth pointing out that in reference [8] there is a misprint in the plus sign within the value of $f_{2}(x)$. Further, it is verified numerically in Table 1 that the (SCA) has fourth order rate of convergence.

\begin{tabular}{|c|c|c|c|c|c|c|}
\hline \multirow[t]{2}{*}{$\mathrm{N}$} & \multicolumn{2}{|c|}{ Max $\operatorname{Err}(\mathrm{SCA})$} & \multicolumn{2}{|c|}{ Order (SCA) } & \multicolumn{2}{|c|}{$\begin{array}{c}\text { Max Err in Geng [8] } \\
\text { with } N=20\end{array}$} \\
\hline & $\mathrm{u}$ & $\mathrm{v}$ & $\mathrm{u}$ & $\mathrm{V}$ & $\mathrm{u}$ & $\mathrm{v}$ \\
\hline 8 & $1.38263(-5)$ & $5.29328(-5)$ & 3.92 & 3.79 & & \\
\hline 16 & $9.16071(-7)$ & $3.83813(-6)$ & 3.97 & 3.97 & $2.2(-3)$ & $8.0(-3)$ \\
\hline 32 & $5.84556(-8)$ & $2.44426(-7)$ & 4.00 & 4.00 & & \\
\hline 64 & $3.65831(-9)$ & $1.53165(-8)$ & & & & \\
\hline
\end{tabular}

Table 1. Numerical solution of Example 1.

The approximate solution using (SCA) and $N=8$ nodal points on a uniform mesh is depicted in Figure 1.

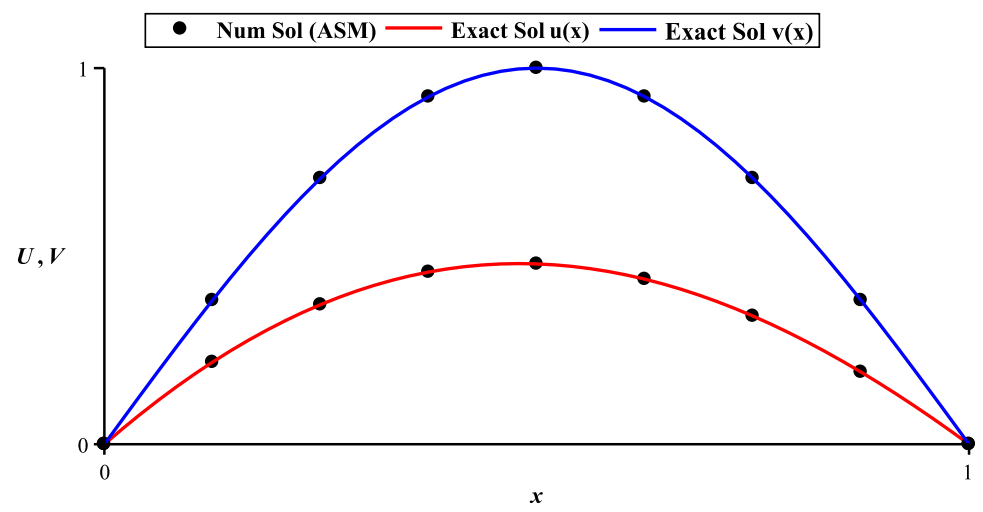

Figure 1. Numerical solution of Example 1 using (SCA) and $N=8$ on uniform mesh.

Example 2. Consider the following nonlinear system which is complimented with boundary conditions:

$$
\left\{\begin{array}{l}
u^{\prime \prime}(x)+x u(x)+2 x v(x)+x u^{2}(x)=f_{1}(x) \\
v^{\prime \prime}(x)+v(x)+x^{2} u(x)+(\sin x) v^{2}(x)=f_{2}(x), \\
u(0)=u(1)=0, \quad v(0)=v(1)=0
\end{array}\right.
$$


where $0 \leq x \leq 1$

$$
\begin{aligned}
& f_{1}(x)=2 x \sin (\pi x)-2+x^{2}-2 x^{4}+x^{5} \\
& f_{2}(x)=(1-x) x^{3}+\left(1-\pi^{2}\right) \sin (\pi x)+\sin x \sin ^{2}(\pi x) .
\end{aligned}
$$

The boundary problem (3.2) has the exact solutions $u(x)=x(1-x)$ and $v(x)=\sin (\pi x)$. This problem has been taken from Geng [8]: we should point out two small misprints in that paper. The first term in $f_{1}$ should be $2 x \sin (\pi x)$, and not $-2 x \sin x$ and in the second equation we should have $v^{\prime \prime}$ as the first term and not $v^{\prime}$. Otherwise the exact solutions are not correct.

The maximum errors obtained by spline collocation approach (SCA) using $N=8,16,32,64,128$ nodal points on a uniform mesh, are reported in Table 2 and are compared again with the results obtained by Geng [8]. It is clear that the maximum errors acquired by the (SCA) are smaller than those given in [8] and that the method is of order 4 .

\begin{tabular}{|c|c|c|c|c|c|c|}
\hline \multirow[t]{2}{*}{$\mathrm{N}$} & \multicolumn{2}{|c|}{ Max $\operatorname{Err}(\mathrm{SCA})$} & \multicolumn{2}{|c|}{ Order (SCA) } & \multicolumn{2}{|c|}{$\begin{array}{c}\text { Max Err in Geng }[8] \\
\text { with } N=20\end{array}$} \\
\hline & $\mathrm{u}$ & $\mathrm{v}$ & $\mathrm{u}$ & $\mathrm{V}$ & $\mathrm{u}$ & $\mathrm{V}$ \\
\hline 8 & $7.50014(-6)$ & $7.04046(-5)$ & 3.76 & 3.82 & & \\
\hline 16 & $5.53168(-7)$ & $4.98959(-6)$ & 3.97 & 3.98 & & \\
\hline 32 & $3.52304(-8)$ & $3.16860(-7)$ & 4.00 & 4.00 & $2.2(-3)$ & $8.2(-3)$ \\
\hline 64 & $2.20777(-9)$ & $1.98474(-8)$ & 4.00 & 4.00 & & \\
\hline 128 & $1.38075(-10)$ & $1.24089(-9)$ & & & & \\
\hline
\end{tabular}

Table 2. Numerical solution of Example 2.

Example 3. Consider the nonlinear system:

$$
\left\{\begin{array}{l}
u^{\prime \prime}(x)-(\sin x) v(x)-u^{\prime}(x) v(x)-\ln v(x)=e^{x}-\cos x+x-1 \\
v^{\prime \prime}(x)-u^{\prime}(x)\left(v^{\prime}(x)\right)^{2}-(\sin x) v^{2}(x)=0 \\
u(0)+v(0)+u^{\prime}(0)+v^{\prime}(0)=3 \\
2 u(0)-v(0)+u^{\prime}(0)+3 v^{\prime}(0)=1 \\
u(1)+v(1)-u^{\prime}(1)+v^{\prime}(1)=\cos 1+\sin 1 \\
u(1)+e^{2} v^{\prime}(1)=\cos 1
\end{array}\right.
$$

where $0 \leq x \leq 1$. Problem (3.3) has the exact solutions $u(x)=e^{x}+\cos x$ and $v(x)=e^{-x}$.

In Table 3, we give the maximum error obtained by the spline collocation approach (SCA) using $N=5,10,20,40,80,160$ nodal points on a uniform mesh and then compare them with those reported by Lang [14], who uses quintic splines instead. Our approach, using only cubic splines on a non-uniform mesh, yielded compatible results. The order of the method is approximately 4 and even exceeds it for smaller values of $N$.

Example 4. Consider the singularly perturbed system of reaction-diffusion 
Table 3. Numerical solution of Example 3.

\begin{tabular}{ccccccccc}
\hline \multirow{2}{*}{$\mathrm{N}$} & \multicolumn{2}{c}{ Max Err (SCA) } & & \multicolumn{2}{c}{ Order (SCA) } & & \multicolumn{2}{c}{ Max Err in Lang [14] } \\
\cline { 2 - 3 } \cline { 7 - 8 } \cline { 7 - 8 } & $\mathrm{u}$ & $\mathrm{v}$ & & $\mathrm{u}$ & $\mathrm{v}$ & & $\mathrm{u}$ & $\mathrm{v}$ \\
\hline 5 & $5.319(-5)$ & $6.112(-5)$ & & 5.63 & 6.07 & & $9.075(-6)$ & $1.038(-5)$ \\
10 & $1.076(-6)$ & $9.121(-7)$ & & 4.52 & 6.07 & & $5.422(-7)$ & $6.213(-7)$ \\
20 & $4.702(-8)$ & $1.361(-8)$ & & 4.12 & 2.66 & & $3.379(-8)$ & $3.850(-8)$ \\
40 & $2.703(-9)$ & $2.148(-9)$ & & 3.86 & 3.68 & & $2.108(-9)$ & $2.402(-9)$ \\
80 & $1.86(-10)$ & $1.68(-10)$ & & 3.92 & 3.88 & & $1.93(-10)$ & $2.26(-10)$ \\
160 & $1.23(-11)$ & $1.14(-11)$ & & & & & \\
\hline
\end{tabular}

equations:

$$
\left\{\begin{array}{l}
\epsilon u^{\prime \prime}(x)-2(1+x)^{2} u(x)+\left(1+x^{3}\right) v(x)=-2 e^{x} \\
v^{\prime \prime}(x)+2 \cos \left(\frac{\pi x}{4}\right) u(x)-2.2 e^{1-x} v(x)=-(10 x+1) \\
u(0)=u(1)=v(0)=v(1)=0
\end{array}\right.
$$

where $0 \leq x \leq 1$. This problem has been studied by Matthews [16]. The thickness of the boundary layer for the perturbed system (3.4) is estimated by using the formula

$$
\sigma=\min \left\{\frac{1}{4}, \sqrt{\frac{\epsilon}{\alpha}} \ln N\right\}, \quad \alpha=\min \left\{2(1+x)^{2}\right\}=2 \quad \text { on } \quad[0,1] .
$$

Note that generally $\alpha$ is the minimum value of the absolute value of the coefficient of the $u$-term which is $-2(1+x)^{2}$ for our case.

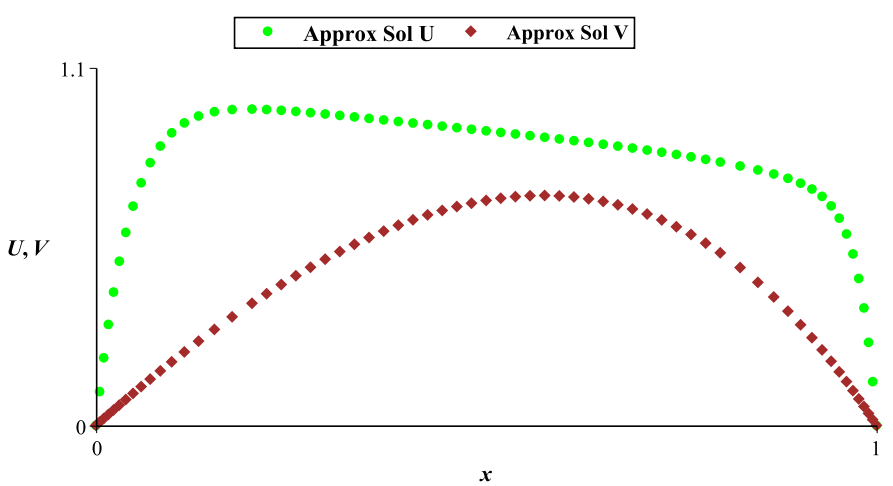

Figure 2. Numerical solution of Example 4 for $\epsilon=2^{-8}$, using (SCA) and $N=64$ on non-uniform mesh.

The solution interval is divided as $[0, \sigma] \cup[\sigma, 1-\sigma] \cup[1-\sigma, 1]$. The number of nodes $N$ is also divided between each part as follows: $N / 4$ on the first and the third subintervals and $N / 2$ nodes in the middle one. After defining the piecewise uniform meshes, we use a mapping/transformation function to redistribute the nodes in such a way to have coarser meshes at the boundaries 
Table 4. Maximum Error of Example 4 for $\epsilon=2^{-8}$.

\begin{tabular}{cccccc}
\hline \multirow{2}{*}{$\mathrm{N}$} & \multicolumn{2}{c}{ Max Err (SCA) } & & \multicolumn{2}{c}{ Max Err in Matthews [16] } \\
\cline { 2 - 3 } \cline { 5 - 6 } & $\mathrm{u}$ & $\mathrm{v}$ & & $\mathrm{u}$ & $\mathrm{v}$ \\
\hline 8 & $7.115(-3)$ & $1.114(-4)$ & & $3.829(-2)$ & $9.091(-3)$ \\
16 & $2.958(-4)$ & $2.116(-5)$ & & $1.713(-2)$ & $2.212(-3)$ \\
32 & $3.886(-5)$ & $2.959(-6)$ & & $7.613(-3)$ & $4.628(-4)$ \\
64 & $5.248(-6)$ & $3.572(-7)$ & & $2.891(-3)$ & $7.838(-5)$ \\
128 & $6.879(-7)$ & $4.655(-8)$ & & $9.955(-4)$ & $1.102(-5)$ \\
\hline
\end{tabular}

and tailored appropriately in order to handle the layer with high accuracy. The approximate solution by the spline collocation approach (SCA) using $N=64$, $\epsilon=2^{-8}$ and non-uniform mesh is illustrated in Figure 2. The maximum errors, using different number of nodes, are reported in Table 4 which shows more accurate approximate solutions obtained by the (SCA) as compared with those results given in [16]. For this problem, the order of the (SCA) reduced to approximately 3 which is a solid indication of the limitation of the method in case of existence of a layer. More precisely, the choice of the mapping redistribution function will definitely affect the order of convergence.

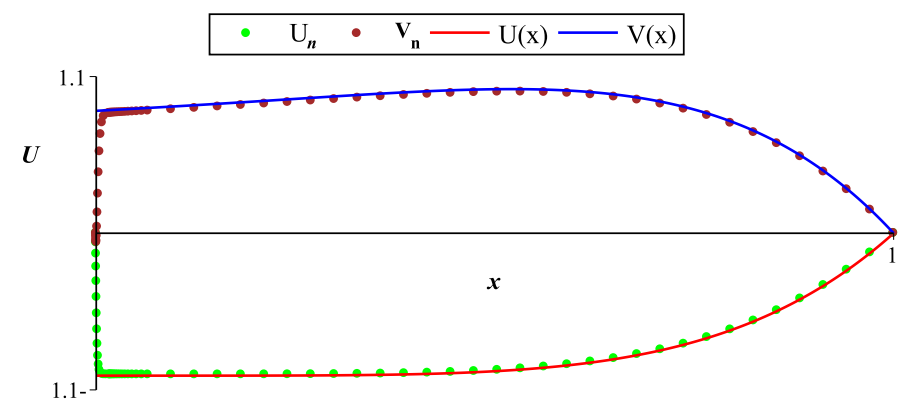

Figure 3. Numerical solution of Example 5 using (SCA) and $N=64$ on non-uniform mesh.

Example 5. In this problem we solve the system of two singularly perturbed convection-diffusion equations:

$$
\left\{\begin{array}{l}
\epsilon_{1} u^{\prime \prime}(x)+3 u^{\prime}(x)=15 x^{4} \\
\epsilon_{2} v^{\prime \prime}(x)+2 v^{\prime}(x)+2.75 u^{\prime}(x)=0.6 e^{x} \\
u(0)=u(1)=v(0)=v(1)=0
\end{array}\right.
$$

where $0 \leq x \leq 1$. System (3.5) was given by Bellew [1], in which the following approximate solutions were manipulated outside the boundary layer that exists at $x=0$ :

$$
u(x)=x^{5}-1 \quad \text { and } \quad v(x)=0.3\left(e^{x}-e\right)-1.375\left(x^{5}-1\right) .
$$


The solution interval is divided as: $\left[0, \sigma_{1}\right] \cup\left[\sigma_{1}, \sigma_{2}\right] \cup\left[\sigma_{2}, 1\right]$, where

$$
\sigma_{1}=2 \frac{\epsilon_{1}}{\gamma_{1}} \ln N, \quad \sigma_{2}=4 \frac{\epsilon_{2}}{\gamma_{2}} \ln N, \quad \gamma_{1}<3, \quad \gamma_{2}<2 .
$$

The number of nodes $N$ is divided within each part as follows: $N / 2$ on the first subinterval and $N / 4$ on each of the second and third. After defining the piecewise uniform meshes, we use a mapping function to redistribute the nodes in such a way so that we have a coarser mesh near the boundary layer. The solution by the spline collocation approach (SCA) using $N=64, \epsilon_{1}=\epsilon_{2}=2^{-8}$ and non-uniform mesh is depicted in Figure 3.

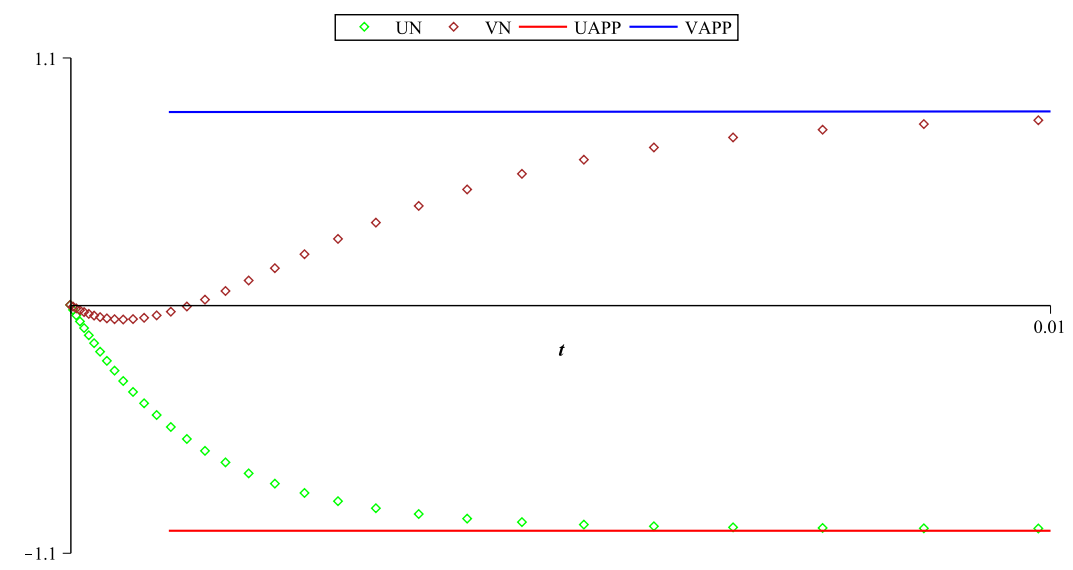

Figure 4. Inner numerical solution and approximate outer solutions given in [1] for Example 5.

Figure 4 shows the numerical solution on the inner region near the boundary layer, together with the approximate solutions suggested by [1]. We note these approximate solutions are not valid within the boundary layer. The maximum residual errors, using $(\mathrm{SCA})$ and different number of nodes, and the order of convergence are compared with numerical solutions given in [1] for $u(t)$ and are presented in Table 5 .

Table 5. Numerical results and comparisons of Example 5.

\begin{tabular}{|c|c|c|c|c|c|c|c|c|c|c|}
\hline \multirow[t]{2}{*}{$\mathrm{N}$} & \multicolumn{4}{|c|}{$\begin{array}{l}\text { Max Err } \\
0 \leq t \leq 0.01\end{array}$} & \multicolumn{4}{|c|}{$\begin{array}{l}\text { Max Err } \\
0.01 \leq t \leq 1\end{array}$} & \multicolumn{2}{|c|}{$\begin{array}{c}\text { Max Err in }[1] \\
0.01 \leq t \leq 1\end{array}$} \\
\hline & $\mathrm{u}$ & $\mathrm{p}$ & $\mathrm{V}$ & Order & $\mathrm{u}$ & Order & $\mathrm{v}$ & Order & $\mathrm{u}$ & Order \\
\hline 64 & $1.58(0)$ & 2.0 & $2.84(0)$ & 2.0 & $3.95(-2)$ & 3.6 & $3.85(-1)$ & 2.8 & $1.74(-1)$ & 0.628 \\
\hline 128 & $4.76(-1)$ & 2.0 & $7.36(-1)$ & 2.0 & $3.28(-3)$ & 3.7 & $5.72(-2)$ & 2.9 & $1.12(-1)$ & 0.751 \\
\hline 256 & $1.22(-1)$ & 2.0 & $1.91(-1)$ & 2.0 & $7.71(-3)$ & 3.7 & $2.59(-4)$ & 3.0 & $6.68(-2)$ & 0.778 \\
\hline 512 & $3.15(-2)$ & & $4.94(-2)$ & & $1.96(-5)$ & & $9.77(-4)$ & & $3.89(-2)$ & 0.830 \\
\hline
\end{tabular}

The results indicate superiority of our suggested approach. As in Example 4 and due to the existence of a boundary layer, we note that the order for such a problem is reduced. 
Example 6. Consider the following system of two singularly perturbed equations:

$$
\left\{\begin{array}{l}
-\epsilon_{1} u^{\prime \prime}(x)-u^{\prime}(x)+2 u^{3}(x)-v(x)=f_{1}(x) \\
-\epsilon_{2} v^{\prime \prime}(x)-2 v^{\prime}(x)+4 v^{2}(x)-u(x)=f_{2}(x) \\
u(0)=u(1)=v(0)=v(1)=0
\end{array}\right.
$$

where $0 \leq x \leq 1$. The exact solution to system (3.6) is given by:

$$
u=\frac{1-e^{-x / \epsilon_{1}}}{1-e^{-1 / \epsilon_{1}}}+\frac{1-e^{-x / \epsilon_{2}}}{1-e^{-1 / \epsilon_{2}}}-2 \sin \left(\frac{\pi x}{2}\right), \quad v=\frac{1-e^{-x / \epsilon_{2}}}{1-e^{-1 / \epsilon_{2}}}-x e^{x-1} .
$$

We omit the values of $f_{1}$ and $f_{2}$ as they are lengthy expressions and can be easily determined by inserting the latter exact solutions into the system (3.6).

For this case, we let $\left\{x_{i}\right\}$ to be a uniform mesh and then we use the redistribution function $w_{i}=\left((1+k)^{x_{i}}-1\right) / k$ in order to have a coarser mesh at $x=a$ by moving the points near the left-hand layer. Since the system in question is nonlinear in $u$ and $v$, we employ Newton's method first in order to linearize the equations. This yields the following iterative scheme:

$$
\begin{aligned}
& -\epsilon_{1} u_{n}^{\prime \prime}(x)-u_{n}^{\prime}(x)+6 u_{n-1}^{2}(x) u_{n}(x)-v_{n}(x)=f_{1}(x)+4 u_{n-1}^{3}, \\
& -\epsilon_{2} v_{n}^{\prime \prime}(x)-2 v_{n}^{\prime}(x)+8 v_{n-1}(x) v_{n}-u_{n}(x)=f_{2}(x)+4 v_{n-1}^{2} .
\end{aligned}
$$

The maximum errors as well as the order are reported in Table 6 which shows the high accuracy of the approximate solutions and the fourth order rate of convergence.

Table 6. Numerical results of Example 6.

\begin{tabular}{ccccc}
\hline $\mathrm{N}$ & Max Err u & Order u & Max Err v & Order v \\
\hline 16 & $2.01754(-3)$ & 4.002 & $1.43152(-3)$ & 3.950 \\
32 & $1.25870(-4)$ & 3.989 & $9.26344(-5)$ & 3.997 \\
64 & $7.92530(-6)$ & 3.999 & $5.80174(-6)$ & 3.999 \\
128 & $4.95382(-7)$ & 4.000 & $3.62784(-7)$ & 4.000 \\
256 & $3.09622(-8)$ & & $2.26766(-8)$ & \\
\hline
\end{tabular}

The numerical solution is shown in Figure 5.

Example 7. Consider the following system of two singularly perturbed equations:

$$
\left\{\begin{array}{l}
-\epsilon y_{1}^{\prime \prime}(x)+2(x+1)^{2} y_{1}(x)-\left(1+x^{3}\right) y_{2}(x)=f_{1}(x) \\
-\epsilon y_{2}^{\prime \prime}(x)-2 \cos \left(\frac{\pi}{4} x\right) y_{1}(x)+2.2 e^{1-x} y_{2}(x)=f_{2}(x) \\
y_{1}(0)=y_{1}(1)=y_{2}(0)=y_{2}(1)=0
\end{array}\right.
$$

where $x \in(0, d) \cup(d, 1)$ and at this $d \in(0,1)$ the functions $f_{1}$ and $f_{2}$ have a single discontinuity. Here

$$
f_{1}(x)=\left\{\begin{array}{ll}
2 e^{x}, & 0 \leq x \leq 0.5, \\
1, & 0.5<x \leq 1,
\end{array} \quad f_{2}(x)= \begin{cases}10 x+1, & 0 \leq x \leq 0.5 \\
2, & 0.5<x \leq 1\end{cases}\right.
$$






Figure 5. Numerical solution of Example 6.

This example has been taken from [19]. This problem has layers at the end points and in the middle of the interval. Labatto redistribution mappings were used on the subintervals $(0,0.5)$ and $(0.5,1)$. For $\epsilon_{1}=\epsilon_{2}=10^{-5}$, the spline collocation approach yields highly aacurate results. Indeed, using the number of nodes $N=64$, the maximum residual errors are $7.0 \times 10^{-24}$ and $2.4 \times 10^{-23}$ for the first and second equations, respectively. In comparison with [19]: the maximum errors obtained are 0.0418 for $u$ and 0.302 for $v$, using the difference between numerical approximations with $N$ and $2 N$ nodes. Figure 6 displays the numerical solutions for this system of equations.

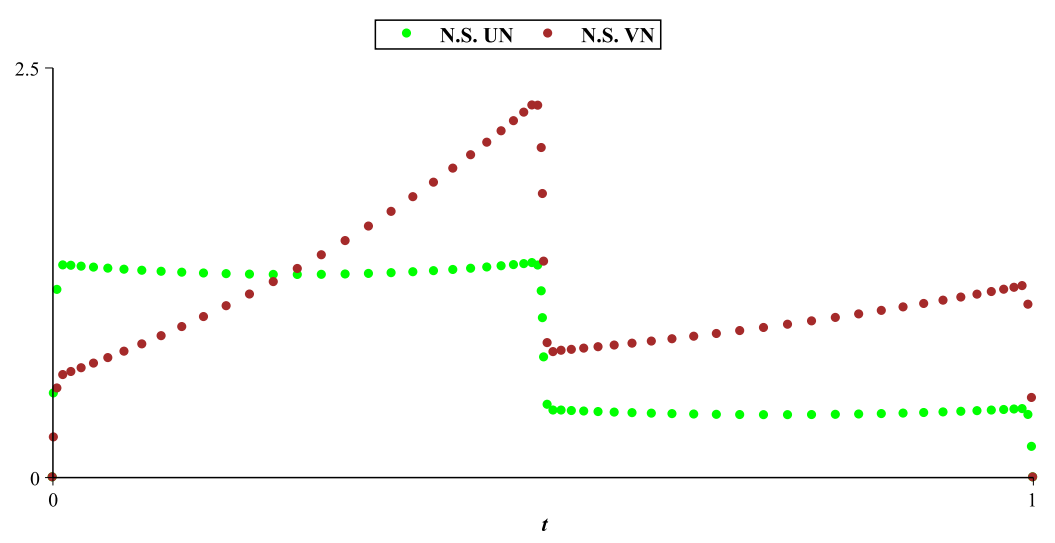

Figure 6. Numerical solution of Example 7. 


\section{Conclusions}

A fourth order cubic spline collocation method have been proposed and discussed for its proper implementation for the numerical solution of an extended class of a system of nonlinear differential equations. The scheme has been successfully applied for both regular systems as well as ones which are singularly perturbed that possess boundary layers. An important factor is that the scheme can handle the boundary layers quite efficiently regardless of its severity. This has been one of the hallmarks of the success of the suggested method which has been exemplified in this article with adequate number of test examples. The numerical results reported in the tables and depicted in the graphs, illustrated the validity of the method and provided highly accurate solutions that are superior when compared with other available methods or compare favorably with them to say the least. The convergence of the suggested strategy is analyzed and it is shown numerically that the rate of convergence is mostly around 4 for regular systems and slightly deteriorates within the layer.

\section{References}

[1] S. Bellew and E. O'Riordan. A parameter robust numerical method for a system of two singularly perturbed convection-diffusion equations. Applied Numerical Mathematics, 51(23):171-186, 2004. http://dx.doi.org/10.1016/j.apnum.2004.05.006.

[2] G.F. Carey and H.T. Dinh. Grading functions and mesh redistribution. SIAM Journal of Numerical Analysis, 22(5):1028-1040, 1985. http://dx.doi.org/10.1137/0722061.

[3] X. Cheng and C. Zhong. Existence of positive solutions for a secondorder ordinary differential system. J. Math. Anal. Appl., 312(1):14-23, 2005. http://dx.doi.org/10.1016/j.jmaa.2005.03.016.

[4] C.C. Christara and K.S. Ng. Adaptive techniques for spline collocation. Computing, 76(3-4):259-277, 2006. http://dx.doi.org/10.1007/s00607-005-0141-3.

[5] C.C. Christara and K.S. Ng. Optimal quadratic and cubic spline collocation on nonuniform partitions. Computing, 76(3-4):227-257, 2006. http://dx.doi.org/10.1007/s00607-005-0140-4.

[6] E.Y. Deeba and S.A. Khuri. Nonlinear equations, pp. 562-570. John Wiley \& Sons, Inc., 1999. http://dx.doi.org/10.1002/047134608X.W2441.

[7] M. Dehghan and A. Saadatmandi. The numerical solution of a nonlinear system of second-order boundary value problems using sinccollocation method. Math. Comput. Modelling, 46(1112):1434-1441, 2007. http://dx.doi.org/10.1016/j.mcm.2007.02.002.

[8] F. Geng and M. Cui. Solving a nonlinear system of second order boundary value problems. J. Math. Anal. Appl., 327(2):1167-1181, 2007. http://dx.doi.org/10.1016/j.jmaa.2006.05.011.

[9] S.A. Khuri and A. Sayfy. Spline collocation approach for the numerical solution of generalized system of second-order boundary-valued problem. Applied Mathematical Sciences J., 3(45):2227-2239, 2009. 
[10] S.A. Khuri and A. Sayfy. A spline collocation approach for the numerical solution of a generalized nonlinear Klein-Gordon equation. Applied Mathematics and Computation, 216(4):1047-1056, 2010. http://dx.doi.org/10.1016/j.amc.2010.01.122.

[11] S.A. Khuri and A. Sayfy. Troesch's problem: A B-spline collocation approach. Mathematical and Computer Modelling, 54(910):1907-1918, 2011. http://dx.doi.org/10.1016/j.mcm.2011.04.030.

[12] S.A. Khuri and A. Sayfy. The boundary layer problem: A fourth-order collocation approach. Computers and Mathematics with Applications, 64(6):2089-2099, 2012. http://dx.doi.org/10.1016/j.camwa.2012.04.005.

[13] S.A. Khuri and A. Sayfy. A spline collocation approach for a generalized parabolic problem subject to non-classical conditions. Applied Mathematics and Computation, 218(18):9187-9196, 2012. http://dx.doi.org/10.1016/j.amc.2012.02.075.

[14] F. Lang and X. Xu. Quintic B-spline collocation method for second order mixed boundary value problem. Computer Physics Communications, 183(4):913-921, 2012. http://dx.doi.org/10.1016/j.cpc.2011.12.017.

[15] J. Lu. Variational iteration method for solving a nonlinear system of secondorder boundary value problems. Comput. Math. Appl., 54(78):1133-1138, 2007. http://dx.doi.org/10.1016/j.camwa.2006.12.060.

[16] S. Matthews, E. O'Riordan and G.I. Shishkin. A numerical method for system of singularly perturbed reaction-diffusion equations. J. Comp. Appl. Math., 145(1):151-166, 2002. http://dx.doi.org/10.1016/S0377-0427(01)00541-6.

[17] A. Saadatmandi, M. Dehghan and A. Eftekhari. Application of He's homotopy perturbation method for non-linear system of second-order boundary value problems. Nonlinear Analysis: Real World Applications, 10(3):1912-1922, 2009. http://dx.doi.org/10.1016/j.nonrwa.2008.02.032.

[18] A. Saadatmandi and J.A. Farsangi. Chebychev finite difference method for a nonlinear system of second-order boundary value problems. Appl. Math. Comput., 192(2):586-591, 2007. http://dx.doi.org/10.1016/j.amc.2007.02.148.

[19] A. Tamilselvan, N. Ramanujam and V. Shanthi. A numerical method for singularly perturbed weakly coupled system of two second order ordinary differential equations with discontinuous source term. Journal of Computational and Applied Mathematics, 202(2):203-216, 2007. http://dx.doi.org/10.1016/j.cam.2006.02.025.

[20] T. Valanarasu and N. Ramanujam. An asymptotic initial value method for boundary value problems for a system of singularly perturbed second order ordinary differential equations. Applied Mathematics and Computation, 147(1):227240, 2004. http://dx.doi.org/10.1016/S0096-3003(02)00663-X. 